\title{
Padrões linguísticos para detecção de ironia em múltiplos idiomas
}

\author{
Diulia J. Deon ${ }^{1}$, Leonardo P. dos Santos ${ }^{2}$, Larissa A. de Freitas ${ }^{1}$ \\ ${ }^{1} \mathrm{CDTec}$ - Universidade Federal de Pelotas (UFPel) \\ Rua Gomes Carneiro, 1 - 96.010-610 - Pelotas - RS - Brasil \\ ${ }^{2}$ Faculdade de Letras - Universidade de São Paulo (USP) \\ Rua da Reitoria, 374 - 05508-220 - São Paulo - SP - Brasil \\ \{djdeon, larissa\}@inf.ufpel.edu.br; leonardosantsperegmail.com
}

\begin{abstract}
Most of studies in Sentiment Analysis aims to classify utterances according to their polarity. Irony represents a challenge to this field, since it comprises the meaning inversion in text. On the other hand, the big volume of data posted on Social Network and the micropost genre characteristics draw researchers' attention. In this paper, we propose to analyze identified linguistic patterns to irony detection in microposts in Portuguese, English, Italian and Spanish.
\end{abstract}

Resumo. Grande parte dos estudos em Análise de Sentimento visam a classificar enunciados de acordo com sua polaridade. A ironia se apresenta como um desafio a essa área, visto que compreende a inversão do sentido no texto. Por outro lado, o grande volume de dados publicados nas Redes Sociais e as características do gênero micropost chamam a atenção dos estudiosos. Nesse artigo, propomos analisar padrões linguísticos identificados para detecção de ironia em microposts em Português, Inglês, Italiano e Espanhol.

\section{Introdução}

Com o crescente uso de blogs, fóruns, wikis e vários outros tipos de mídias sociais, os indivíduos comuns se tornaram protagonistas no mercado da comunicação. Com isso, essas plataformas se tornaram grandes fontes de informação. Ao passo que as pessoas começaram a compartilhar experiências e opiniões sobre produtos, serviços e experiências na Web, a mesma passou a compor um repositório cuja avaliação manual tornou-se inviável devido ao enorme volume de dados. Apenas no Twitter, a média de publicações diárias é próxima de 500 milhões [Aslam 2019].

Devido ao exorbitante volume de dados e à publicação voluntária de opiniões e avaliações de produtos e serviços na rede, surgiu o interesse da indústria e da comunidade científica no processamento automático do conteúdo publicado na Web. Nesse sentido, atualmente, os cientistas se organizam em campos do conhecimento voltados ao desenvolvimento de técnicas para esse fim, como é o caso da Análise de Sentimento.

De acordo com Pang (2018) [Pang and Lee 2008], a Análise de Sentimento preocupa-se em definir a opinião que um documento expressa sobre determinado tópico. As opiniões podem representar impactos positivos/favoráveis ou negativos/desfavoráveis 
e se tornam relevantes devido ao hábito humano de consultar opiniões alheias antes de tomar decisões.

Todavia, apesar dos avanços no desenvolvimento tecnológico em Inteligência Artificial, o processamento automático de opinião se depara com vários obstáculos. Dentre eles, destaca-se a linguagem figurada. Nesse ínterim, a ironia se mostra como um desafio ao Processamento de Língua Natural. Em Análise de Sentimento, considera-se que o primeiro passo para o processamento de textos irônicos é a detecção automática desse fenômeno linguístico. Diversos estudos (como [Carvalho et al. 2009], [Gonzales-Ibanez et al. 2011], [Reyes et al. 2013], [Freitas et al. 2014], [Ortega-Bueno et al. 2019] e [Ghanem et al. 2019]) apresentam métodos baseados em regras linguística e aprendizado de máquina capazes de identificar ironia em diferentes idiomas. Em geral, tweets e outros textos curtos compõem corpora para esse tipo de trabalho.

Diante desses motivos, o presente estudo visa a testar e avaliar a implementação de padrões linguísticos aplicados a textos escritos em Português, Inglês, Italiano e Espanhol. Este artigo está estruturado da seguinte forma: a segunda seção apresenta concepções de ironia; a terceira seção apresenta trabalhos correlatos; a quarta seção apresenta nossa proposta de trabalho; a quinta seção apresenta os resultados obtidos e, por fim, a última seção apresenta as considerações finais e trabalhos futuros.

\section{Concepções sobre Ironia}

Usualmente, compreende-se ironia como um recurso linguístico utilizado com a finalidade de expressar o sentido oposto ao literal de um enunciado [Cignarella et al. 2018]. Seres humanos tem relativa facilidade para compreender ironias, já que os mesmos conhecem o contexto do enunciado. Todavia, há estudos que defendem a ideia que essa tarefa é complicada não apenas para os sistemas, mas também para os humanos [Maynard and Greenwood 2014].

Apesar de muitos pesquisadores projetarem seus esforços em estudos em ironia, não há consenso acerca de definição desse fenômeno linguístico. [Searle 1969] e [Grice 1975] compreendem ironia como uma aparente violação de princípios pragmáticos. Ainda assim, ironia parece ser um mecanismo mais complexo. [Reyes et al. 2012] define ironia como uma propriedade contraditória em determinado contexto ou evento. [Kreuz and Glucksberg 1989] afirma que a presença de ironia transmite um significado pragmático ao aludir a expectativas (falhas ou não).

Ao investigar sobre ironia, é comum que nos deparemos com discussões acerca das diferenciações (ou não) entre ironia e sarcasmo. Há estudos que optam por diferenciar e escolher o termo mais adequado entre ambos, como [Grice 1975] [Sperber and Wilson 1981], enquanto outros consideram sarcasmo e ironia o mesmo fenômeno, não fazendo distinções, como [Attardo 2000], [Reyes et al. 2013] e [Van Hee et al. 2016]. [Gibbs and Colston 2001] defende que o sarmasmo, aliado à outros recursos linguísticos como humor, hipérbole e retórica, pode ser considerado um tipo de ironia. [Marchetti et al. 2007] apresenta a ironia como um conceito-guarda-chuva, isto é, um conceito que inclui outros subconceitos (nesse caso, o sarcasmo e a sátira, por exemplo).

Levando em conta a maior parte dos estudos em Linguística Computacional e 
Análise de Sentimento, optamos por adotar o termo ironia, sem fazer distinções entre ironia, sarcasmo e eventuais outros conceitos. Vale salientar que, ao longo do presente artigo, ressalvas são feitas devido a menções de outros trabalhos.

\section{Trabalhos Correlatos}

O interesse da comunidade acadêmica em descrever e identificar o uso de ironia em textos escritos vêm crescendo nos últimos anos. $\mathrm{Na}$ literatura disponível, é possível encontrar diversos trabalhos relacionados a essa temática, tais como: [Carvalho et al. 2009], [Gonzales-Ibanez et al. 2011], [Reyes et al. 2013], [Freitas et al. 2014], [Ortega-Bueno et al. 2019] e [Ghanem et al. 2019].

Carvalho et al. (2009) apresentaram pistas para detecção de ironia baseadas em emoticos, onomatopéias, pontuações e uso de citações. Gonzales-Ibalnez et al. (2011) apontaram que os contextos (nível pragmático de análise linguística) são extremamentes relevantes, sendo os aspectos lexicais insuficientes para a detecção de ironia. Reyes et al. (2013) propuseram uma detecção automática de ironia a partir da aplicação de técnicas que se valem de padrões linguísticos, como n-gramas e part of speech n-gramas. Freitas et al. (2014) estabeleceram 13 padrões linguísticos em um corpus de tweets em Português Brasileiro a partir de um contexto pré-definido, já que, segundo os autores, é importante considerar as expectativas dos falantes para compreender os enunciados.

Além desses, nesse campo de investigação científica, existem competições nas quais pesquisadores desenvolvem métodos para detecção de ironia. Exemplos dessas competições são a Evalita ${ }^{1}$ (sigla do inglês Evaluation of Natural Language Processsing and Speech Tools for Italian), a SemEval ${ }^{2}$ (sigla do inglês International Workshop on Semantic Evaluation), a IroSvA ${ }^{3}$ (sigla do inglês Irony Detection in Spanish Variants) e a $\operatorname{IDAT}^{4}$ (sigla do inglês Irony Detection in Arabic Tweets). Nestas competições, há tarefas diretamente relacionadas à detecção de ironia.

Cignarella et al. (2018) apresentam os resultados da edição de 2018 do Evalita, especificamente na Tarefa de Detecção de Ironia em Tweets em Italiano (IronITA). Essa tarefa incluiu duas semi-tarefas: (i) detecção de ironia - task $A$ e (ii) detecção de diferentes tipos de ironia, com destaque para o sarcasmo - task $B$. Nessa competição, 17 equipes participaram da task $A$ e 7 equipes participaram da task $B$.

Van Hee et al. (2018) explanam a primeira edição da atividade compartilhada de detecção de ironia no SemEval. A tarefa de Detecção de Ironia em Tweets em Inglês recebeu submissões de 43 equipes para a task $A$ e 31 equipes para a task $B$.

Ortega-Bueno et al. (2019) apresentam os resultados da primeira edição da atividade compartilhada de detecção de ironia em Espanhol, denominada IroSvA. A tarefa é composta por três subtarefas diferentes: (i) detecção de ironia em tweets da Espanha, (ii) detecção de ironia em tweets mexicanos e (iii) detecção de ironia em notícias comentários de Cuba. Nessa competição 12 equipes participaram simultaneamente nas três subtarefas.

Ghanem et al. (2019) apresentam os resultados do IDAT (do inglês Irony Detec-

\footnotetext{
${ }^{1}$ http://www.evalita.it/

${ }^{2}$ http://alt.qcri.org/semeval2019/

${ }^{3}$ http://www.autoritas.net/IroSvA2019/

${ }^{4}$ https://www.irit.fr/IDAT2019/
} 
tion in Arabic Tweets). A tarefa consiste em uma classificação binária de tweets como irônicos ou não irônicos. Nessa competição 18 equipes participaram. Os métodos dos participantes variaram de técnicas clássicas de aprendizado de máquina até métodos de ensemble.

\section{Proposta}

Neste trabalho, aplicamos padrões linguísticos de ironia em corpora contendo microposts escritos em quatro idiomas.

\subsection{Corpora}

Quatro corpora distintos foram utilizadas neste trabalho. Os corpora dos idiomas Inglês, Italiano e Espanhol fizeram parte de competições envolvendo a tarefa de detecção de ironia nos anos de 2018 e 2019. O corpus do idioma Português Brasileiro faz parte de um trabalho de conclusão de curso datado de 2018 [Silva 2018].

Os corpora estão descritos a seguir.

- Português Brasileiro: A base de dados para detecção de ironia em Português Brasileiro é composta por 12.700 tweets potencialmente irônicos, contendo, obrigatoriamente, as hashtags \#ironia e \#sarcasmo, postados entre 08/10/2014 e 10/06/2017 [Silva 2018]; 2.700 tweets potencialmente não irônicos de cunho geral [Silva 2018] e 804 tweets com a expressão "Fim do mundo", anotados manualmente como irônicos e não irônicos, postados entre 19/12/2012 e 23/12/2012 [Freitas et al. 2014].

- Inglês - SemEval 2018: A base de dados da Task3 do SemEval 2018 [Van Hee et al. 2018] é composta por 3.834 tweets, contendo tweets com as hashtags \#irony, \#sarcasm e \#not. Todos os tweets foram coletados entre 01/12/2014 e 04/01/2015 [Van Hee et al. 2016]. Os tweets foram anotados como irônicos ou não irônicos e, ainda, classificados em diferentes tipos de ironia: ironia verbal, situação irônica e não irônica.

- Italiano - IronITA 2018: A base de dados do IronITA 2018 [Cignarella et al. 2018] é composta por 3.977 tweets distribuídos em 2.023 irônicos e 1.957 não irônicos. Os tweets foram anotados de acordo com diferentes tipos de ironia: sarcástico, não sarcástico e não irônico.

- Espanhol - IroSvA 2019: A base de dados do IroSvA 2019 [Ortega et al. 2019] consiste em 9.000 mensagens curtas sobre diferentes tópicos escritos em Espanhol (3.000 de Cuba, 3.000 do México e 3.000 da Espanha) anotados como irônicos e não irônicos.

\subsection{Padrões Linguísticos de Ironia}

Sete padrões foram implementados e utilizados neste trabalho. Os padrões envolvem o uso de listas, expressões exatas e símbolos, conforme descritos abaixo (Tabela 1).

- P1: uso de risadas. Expressões de riso encontradas na $\mathrm{Web}^{5}$ foram utilizadas nesse padrão. Assim como os trabalhos de [Carvalho et al. 2009] e [Freitas et al. 2014] assumimos que textos com expressões de riso são irônicos.

\footnotetext{
${ }^{5}$ https://awebic.com/humor/como-internautas-riem/
} 
Tabela 1. Padrões Linguísticos.

\begin{tabular}{|c|c|}
\hline Padrões & Expressões \\
\hline P1 & Risadas \\
\hline P2 & Emoticons \\
\hline P3 & Expressão Exata \\
\hline P4 & $\ldots<$ Expressão $>!$ \\
\hline P5 & Letra Maiúscula \\
\hline P6 & $*_{i}\left|*_{i}\right| !^{*}\left|?^{*}\right| i^{*} i^{*}\left|i^{*} i^{*}\right| !^{*} ?^{*} \mid ?^{*} ! *$ \\
\hline P7 & “Expressão $>$ " \\
\hline
\end{tabular}

- P2: uso de emoticons. Emoticons expressam sentido humorístico. Por isso, identificamos que todas as ocorrências com estes símbolos podem representar ironia.

- P3: uso de expressões exatas que já foram consolidadas. As expressões exatas demostram que o enunciado não significa o que literalmente está escrito em palavras. Por isso, identificamos que estas expressões exatas podem representar uma declaração irônica. Para cada idioma de análise usamos as seguintes expressões exatas: "só que (não)", "sim,", "na boa" - Português Brasileiro; "not" - Inglês; "solo che non è" - Italiano; "no te creas","no es cierto", "es broma" - Espanhol.

- P4: uso de uma expressão entre reticências e ponto de exclamação. Declarações irônicas são escritas após reticências e antes do ponto de exclamação, como se o autor do texto curto quisesse acrescentar um comentário que expressasse sua opinião sobre o que acabou de ser dito.

- P5: uso de letra maiúscula. Os textos ou trechos de textos escritos em maiúscula são utilizados para destacar/dar ênfase a significados figurativos, como os irônicos.

- P6: uso de pontuação repetida. A pontuação transmite um conteúdo adicional ao enunciado. Usando ponto de exclamação e de interrogação, as emoções podem ser comunicadas e muitas vezes podem ser pistas para mensagens humorísticas. A repetição do ponto de exclamação e de interrogação e a combinação entre eles, como “!!!”, “????”, “!!!!???”, podem ser pistas para conteúdo irônico. Já que um dos idiomas de análise é o Espanhol, acrescentamos no padrão as pontuações “i”e " $i$ ".

- P7: uso de uma expressão entre aspas. As aspas além de transcreverem um texto já escrito, são utilizadas para destacar certas expressões e servem para dar ênfase a significados figurativos, como os irônicos.

\section{Resultados}

Ao aplicarmos os padrões linguísticos na base de dados de [Freitas et al. 2014] e [Silva 2018], obtivemos os seguintes resultados para dectecção de ironia: Tabela 2. O total é de 14757 tweets, já que, utilizamos os dados de treino sem repetições. Como é possível observar na Matriz de Confusão do Português, a Revocação é de 41\%, a Precisão é de $34 \%$ e a de Medida-F é de $34 \%$.

Ainda, após aplicarmos os mesmos padrões linguísticos na base de dados do SemEval 2018 (dados de treino task A com emoji e hashtags de ironia), obtivemos os seguintes resultados para detecção de ironia: Tabela 3. Como apresenta a Matriz de Confusão do Inglês, a Revocação é de $35 \%$, a Precisão é de $49 \%$ e a de Medida-F é de $41 \%$. 
Tabela 2. Matriz de Confusão do Português.

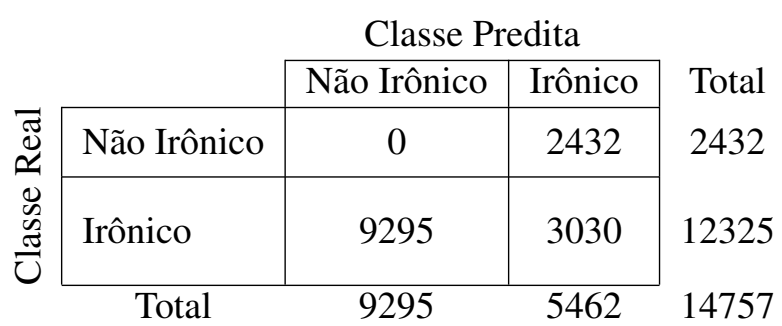

Tabela 3. Matriz de Confusão do Inglês.

\begin{tabular}{|c|c|c|c|c|}
\hline & & \multicolumn{2}{|c|}{ Classe Predita } & \multirow[b]{2}{*}{ Total } \\
\hline & & Não Irônico & Irônico & \\
\hline \multirow{2}{*}{ 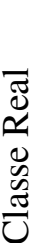 } & Não Irônico & 1228 & 695 & 1923 \\
\hline & Irônico & 1239 & 672 & 1911 \\
\hline & Total & 2467 & 1367 & $38 ?$ \\
\hline
\end{tabular}

Os resultados da detecção de ironia através dos padrões linguísticos na base de dados do IronITA 2018 são apresentados na Tabela 4. Como podemos observar na Matriz de Confusão do Italiano, a Revocação é de $34 \%$, a Precisão é de $47 \%$ e a de Medida-F é de $40 \%$.

Tabela 4. Matriz de Confusão do Italiano.

\begin{tabular}{|c|c|c|c|c|}
\hline & \multicolumn{2}{|c|}{ Classe Predita } & \multirow{3}{*}{$\begin{array}{l}\text { Total } \\
1954\end{array}$} \\
\hline & & Não Irônico & Irônico & \\
\hline & Não Irônico & 1175 & 779 & \\
\hline & Irônico & 1320 & 703 & 2023 \\
\hline & Tota & 2495 & 1482 & 977 \\
\hline
\end{tabular}

Por fim, ao aplicarmos os padrões linguísticos na base de dados do IroSvA 2019, obtivemos os seguintes resultados para detecção de ironia: Tabela 5 (a), (b) e (c). É importante salientar que o total é 2400 mensagens curtas, uma vez que utilizamos os dados de treino sem repetições. De acordo com o que observa-se na Matriz de Confusão do Espanhol, a Revocação é de $41 \%$ para Cuba, $35 \%$ para a variedade do México, $30 \%$ para a variedade da Espanha; a Precisão é de 34\% para a variedade de Cuba, 32\% para a variedade do México e para a variedade Espanha; a Medida-F é de $37 \%$ para a variedade cubana, $33 \%$ para a variedade mexicana e $31 \%$ para a variedade espanhola.

A partir dos resultados obtidos, podemos observar que padrões relacionados à linguagem simbólica, como expressões de riso (P1) e emoticons (P2), são os melhores indícios de ironia e sarcasmo para o idioma Português Brasileiro. Além disso, o padrão relacionado a expressões exatas (P3) provou ser uma boa escolha para os idiomas Português e Inglês , pois "só que (não)" e "not" são culturalmente considerados irônicos.

O desempenho dos sete padrões linguísticos é significativo para a detecção de 
Tabela 5. Matriz de Confusão do Espanhol

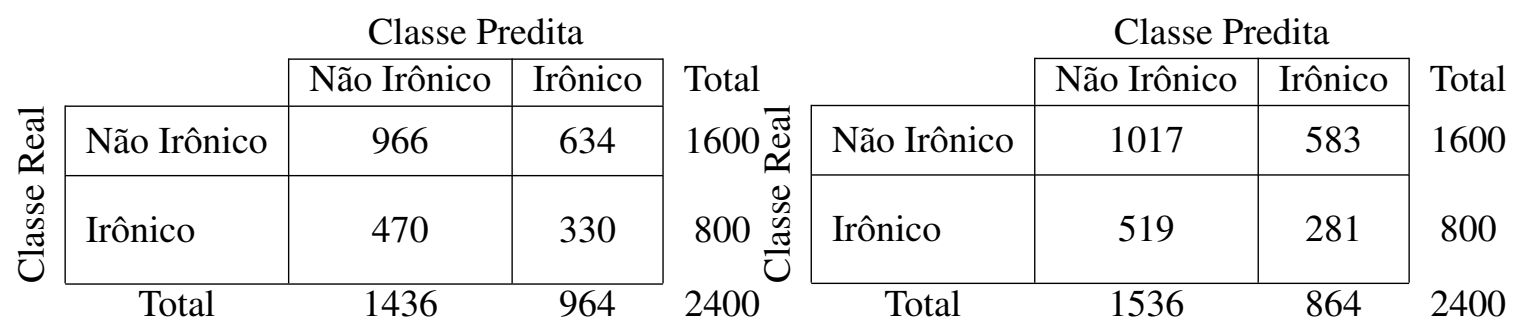

(a) Espanhol de Cuba.

(b) Espanhol do México.

\begin{tabular}{|c|c|c|c|c|}
\hline & \multicolumn{3}{|c|}{ Classe Predita } \\
\hline & & Não Irônico & Irônico & Total \\
\hline$\overline{\mathscr{D}}$ & Não Irônico & 1094 & 506 & 1600 \\
\hline 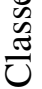 & Irônico & 557 & 243 & 800 \\
\hline & Total & 1651 & 749 & 0 \\
\hline
\end{tabular}

(c) Espanhol da Espanha.

ironia em múltiplos idiomas. Acreditamos que o valor de revocação $(0.30$ - 0.41$)$ é um bom resultado, considerando que é um sistema baseado em padrões. Com os dados de teste da competição IroSvA obtivemos uma Medida-F de 53\% [Ortega-Bueno et al. 2019]. O sistema que obteve melhor resultado obteve uma Medida-F de $68 \%$ e é baseado em um modelo Transformers Encoders.

\section{Considerações Finais e Trabalhos Futuros}

Conforme defendido ao longo do presente texto, declarações irônicas requerem operações mais complexas do que simplesmente interpretar o significado oposto de uma sentença. Embora seja este um estudo inicial, acreditamos que podemos oferecer resultados relevantes no estabelecimento desses sete padrões linguísticos na detecção de ironia em múltiplos idiomas.

O presente artigo apresentou, portanto, um panorama do estado da arte em detecção de ironia em microposts em quatro idiomas (Português, Espanhol, Italiano e Inglês), com ênfase nos padrões linguísticos. Ao total, sete padrões linguísticos foram testados sobre quatro corpora nesses idiomas. A partir dessas aplicações, realizamos uma análise dos resultados. Com isso, foi possível observar que o desempenho dos sete padrões linguísticos foi significativo para a tarefa detecção de ironia nos idiomas supracitados.

Como trabalhos futuros, elencamos três itens centrais pertinentes à detecção de ironia: (i) implementação de técnicas de Deep Learning; (ii) aplicação dos padrões linguísticos apresentados em outros idiomas, por fim, (iii) avaliar o impacto da ironia na tarefa de análise de sentimento.

Considerando o estado da arte neste campo de investigação, observa-se que as técnicas de Deep Learning têm ganhado cada vez mais visibilidade, tendo em vista que seus resultados têm se apresentado incrivelmente competitivos e precisos. Desse modo, acreditamos que a implementação dessas técnicas é promissora na detecção de ironia, podendo apresentar significativos resultados e avanços para investigações nesse sentido. 
Ademais, além da aplicação dos padrões linguísticos em Português, Inglês, Italiano e Espanhol, pretendemos realizar essa avaliação também em outros idiomas. Nesse caso, especificamente, projetamos desenvolver a investigação sobre tweets escritos em Árabe (base de dados para detecção de ironia da IDAT 2019).

Além disso, pretendemos avaliar o impacto da ironia na tarefa de Análise de Sentimento. Como já mencionado aqui, a ironia nos enunciados se apresenta como um obstáculo aos estudos em Análise de Sentimento. Todavia, até o momento, não nos dedicamos a avaliar e mapear, levantando dados quantitativos, os impactos desse fenômeno linguístico nesse processo analítico em múltiplos idiomas.

\section{Referências}

Aslam, S. (2019). Twitter statistics. https://www.omnicoreagency.com/ twitter-statistics/. Acessado em: 25-05-2019.

Attardo, S. (2000). Irony as relevant inappropriateness. Pragmatics, 32(6):793-826.

Carvalho, P., Sarmento, L., and Silva, M. J. (2009). Clues for detecting irony in usergenerated contents: oh...!! it's "so easy";-). In 1st International CIKM Workshop on Topic-sentiment Analysis for Mass Opinion, pages 53-56.

Cignarella, A., Frenda, S., Basile, V., Bosco, C., Patti, V., and Rosso, P. (2018). Overview of the evalita 2018 task on irony detection in italian tweets. In 6th Evaluation Campaign of Natural Language Processing and Speech Tools for Italian.

Freitas, L. A., Vanin, A., Hogetop, D., Bochernitsan, M., and Vieira, R. (2014). Pathways for irony detection in tweets. In 29th Symposium on Applied Computing, pages 628633.

Ghanem, B., Karoui, J., Benamara, F., Moriceau, V., and Rosso, P. (2019). Idat@ fire2019: Overview of the track on irony detection in arabic tweets. In Working Notes of FIRE 2019 - Forum for Information Retrieval Evaluation, Kolkata, India, December 12-15, 2019, volume 2517 of CEUR Workshop Proceedings, pages 380-390. CEUR-WS.org.

Gibbs, R. W. and Colston, H. L. (2001). The risks and rewards of ironic communication. IOS Press.

Gonzales-Ibanez, R., Muresan, S., and Wacholder, N. (2011). Identifying sarcasm in twitter: a closer look. In 49th Annual Meeting of the Association for Computational Linguistics: Human Language Technologies, pages 581-586.

Grice, H. P. (1975). Logic and conversation. Academic Press.

Kreuz, R. J. and Glucksberg, S. (1989). How to be sarcastic: The choice reminder theory of verbal irony. Experimental Psychology, 118(4):374-386.

Marchetti, A., Massaro, D., and Valle, A. (2007). Non dicevo sul serio. Riflessioni su ironia e psicologia. Franco Angeli.

Maynard, D. and Greenwood, M. A. (2014). Who cares about sarcastic tweets? investigating the impact of sarcasm on sentiment analysis. In 9th International Conference on Language Resources and Evaluation. 
Ortega, R., Rangel, F., Hernández, I., Rosso, P., Montes, M., and Pagola, M. (2019). Overview of the task on irony detection in spanish variants. In 34th Conference of the Spanish Society for Natural Language Processing.

Ortega-Bueno, R., Rangel, F., Farías, D. I. H., Rosso, P., y Gómez, M. M., and MedinaPagola, J. (2019). Overview of the task on irony detection in spanish variants. In Proceedings of the Iberian Languages Evaluation Forum co-located with 35th Conference of the Spanish Society for Natural Language Processing, IberLEF@SEPLN 2019, Bilbao, Spain, September 24th, 2019, volume 2421 of CEUR Workshop Proceedings, pages 229-256. CEUR-WS.org.

Pang, B. and Lee, L. (2008). Opinion mining and sentiment analysis. Foundations and Trends in Information Retrieval, 2:1-135.

Reyes, A., Rosso, P., and Buscaldi, D. (2012). From humor recognition to irony detection: The figurative language of social media. Data and Knowledge Engineering, 74:2-12.

Reyes, A., Rosso, P., and Veale, T. (2013). A multidimensional approach for detecting irony in twitter. Language Resources and Evaluation, 47(1):239-268.

Searle, J. R. (1969). Speech Acts: An Essay in the Philosophy of Language. Cambridge University Press.

Silva, F. R. (2018). Detecção de ironia e sarcasmo em língua portuguesa: uma abordagem utilizando deep learning. https://github.com/fabio-ricardo/ deteccao-ironia. Acessado em: 25-05-2019.

Sperber, D. and Wilson, D. (1981). Irony and the Use - Mention Distinction. Academic Press.

Van Hee, C., Lefever, E., and Hoste, V. (2016). Guidelines for annotating irony in social media text, version 2.0. Technical report, LT3 Technical Report Series.

Van Hee, C., Lefever, E., and Hoste, V. (2018). Semeval-2018 task 3: Irony detection in english tweets. In 12th International Workshop on Semantic Evaluation. 